\title{
Repetitive rebrightening of EG Cancri: Evidence for viscosity decay in the quiescent disk?
}

\author{
Y. Osaki ${ }^{1}$, F. Meyer ${ }^{2}$, and E. Meyer-Hofmeister ${ }^{2}$ \\ 1 Faculty of Education, Nagasaki University, Nagasaki 852-8521, Japan \\ 2 Max-Planck-Institut für Astrophysik, Karl-Schwarzschild Str. 1, 85740 Garching, Germany
}

Received 22 December 2000 / Accepted 12 February 2001

\begin{abstract}
A WZ Sge-type dwarf nova, EG Cancri, exhibited six consecutive mini-outbursts with a mean interval of about seven days after the end of the main outburst in 1996/1997. Most unusual was that the star abruptly entered into a deep faint minimum after such frantic activities. We propose that this peculiar phenomenon may be understood in terms of viscosity decay in the cold disk. In this picture, the viscosity is produced by MHD turbulence due to the magneto-rotational instability ("Balbus-Hawley instability") and dies down exponentially with time when the disk becomes cold because the magnetic fields decay due to finite conductivity in the cold disk (Gammie \& Menou 1998). But the viscosity is refreshed to a high value every time when a mini-outburst occurs (i.e., the disk becomes hot again). It is argued that a sudden cessation of repetitive mini-outbursts may be brought about by a very small reduction in viscosity or a small increase in its decay rate, which may in turn be produced most likely by stochastic fluctuations of magnetic fields. Numerical simulations based on a simple model reproduce the observed light curve of EG Cancri very well. We discuss possible causes why the reflares after the main outburst occur mostly in the WZ Sge-type stars.
\end{abstract}

Key words. accretion disks - cataclysmic variables - instabilities - magnetic fields - stars individual: EG Cnc, WZ Sge

\section{Introduction}

WZ Sge stars are a small group of dwarf novae, exhibiting large-amplitude outbursts (6-8 mag instead of $2-5 \mathrm{mag}$ ) and very long recurrence times (decades instead of months), compared with typical dwarf novae (Bailey 1979; Downes 1990; O'Donoghue et al. 1991). They also belong to the SU UMa-type sub-class of dwarf novae, which exhibit the two different types of outbursts, a short "normal outburst" (with a typical outburst duration of a few days) and a long "superoutburst" lasting for more than ten days. The superoutbursts are almost always accompanied by "superhumps". An appearance of superhumps during an outburst is taken as evidence for a SU UMa-type dwarf nova. These superhumps are periodic humps in the photometric light curve with periods slightly longer than the orbital period of the binary.

EG Cancri, a WZ Sge-type dwarf nova, underwent a large outburst in December 1996 after 19 years of dormancy (Matsumoto et al. 1998b; Patterson et al. 1998). The WZ Sge-type stars often show rebrightening or reflares after the end of the main outburst (Richter 1992),

Send offprint requests to: Y. Osaki, e-mail: osaki@net.nagasaki-u.ac.jp e.g., in the 1978 outburst of WZ Sge (Patterson et al. 1981), the 1961 outburst of AL Com and the 1995 outburst of the same star (Kato et al. 1996; Howell et al. 1996; Patterson et al. 1996). Thus EG Cnc was closely watched by many observers. EG Cnc in fact made reflares after the end of the main outburst. However, what was unexpected is that EG Cnc made reflares not only once but exhibited six consecutive mini-outbursts with a mean interval of 7 days. The most unusual feature was that the star abruptly stopped the outbursts after the sixth mini-outburst and entered into a deep faint minimum. The light curve of EG Cnc can be found from VSNET (http://www.kusastro.kyoto-u.ac.jp) or in the papers of Kato et al. (1997), Matsumoto et al. (1998a) and Patterson et al. (1998). Figure 1 shows the eruption light curve as obtained by Patterson et al. (1998) from all available data.

As for theory dwarf nova outbursts are now well understood in terms of the disk instability model (see reviews of Cannizzo 1993; Osaki 1996) in which the accretion disk undergoes a thermal relaxation oscillation between a hot ionized state (corresponding to outburst) and a cold unionized state (corresponding to quiescence). Based on the disk instability model, outburst light curves of dwarf 


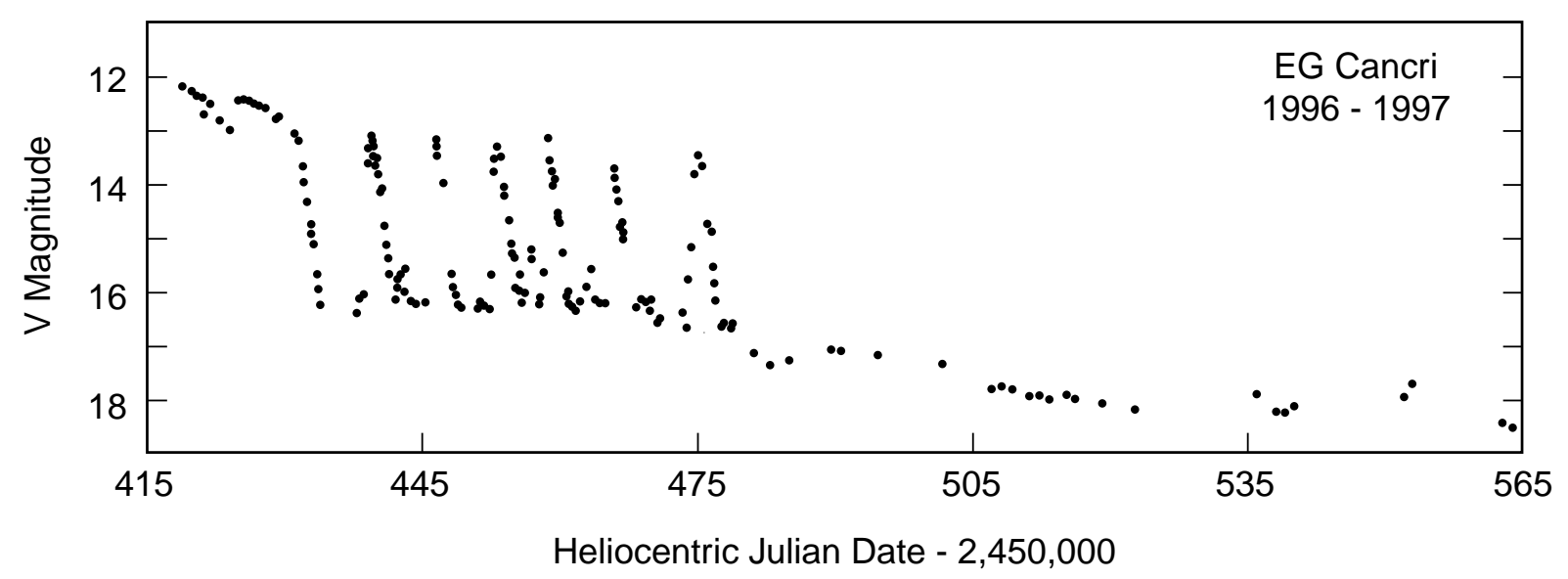

Fig. 1. Outburst light curve of EG Cnc from Patterson et al. (1998)

novae have been simulated by various workers using an $\alpha$ parametrization (Shakura \& Sunyaev 1973) for the "turbulent" viscosity. It has, however, long been recognized (Smak 1984) that we must choose different values for viscosity $\alpha_{\text {hot }}$ in the hot state and $\alpha_{\text {cold }}$ in cold state, i.e., something like $\alpha_{\text {hot }} \sim 0.2$ and $\alpha_{\text {cold }} \sim 0.02$. It has, however, been pointed out by various investigators (Smak 1993; Osaki 1994; 1996, Howell et al. 1995; Meyer et al. 1998) that in the case of WZ Sge stars an extremely low viscosity in quiescence, as low as $\alpha_{\text {cold }} \sim 0.001$ is required to explain their long recurrence times.

The magneto-hydrodynamic origin of the turbulent viscosity in the accretion disk has long been suspected. This idea was put on firmer ground with analysis and recent results of computations for the magneto-rotational instability (Balbus \& Hawley 1991; Brandenburg et al. 1995; Hawley et al. 1996; Armitage 1998). Originally this instability was discovered by Velikov (1959) and Chandrasekhar (1961). Gammie \& Menou (1998) suggested that in the quiescence the MHD turbulence could decay because of the poor conductivity of the cold disk. Meyer \& Meyer-Hofmeister (1999) suggested the following picture for the viscosities in the hot and cold state. The magneto-rotational instabilty of small-scale magnetic fields in the hot disk produce the high viscosity. In quiescence this is not possible anymore (Gammie \& Menou 1998), but the magnetic fields reaching over from the companion star allow a weaker MHD turbulence and cause the lower viscosity in the cold disk. The extreme low viscosity in WZ Sge stars then may be due to the absence of magnetic fields from the companion star in the case of cool degenerate secondary stars.

For the explanation of the repetitive rebrightening of EG Cnc, a few possibilities were suggested. The first is that the observed six mini-outbursts were definitely of the "normal outburst" variety as indicated by the observational evidence (i.e., duration of outburst, spectroscopic evidence, and no photometric evidence of superhumps). Osaki et al. (1997) suggested that mini-outbursts could be produced by a temporal enhancement of viscosity in the quiescent disk just after the main outburst. They demonstrated that the mini-outbursts and their sudden cessation observed in EG Cnc could be reproduced if the viscosity in the quiescent disk was kept high just after the main outburst for the time of the mini-outbursts but then suddenly dropped to the extremely low value of the pre-outburst stage. However, no physical explanation was offered why the viscosity in the quiescent disk of EG Cnc varied in such a way.

Another possibility might be a temporal enhancement of the mass transfer rate from the secondary star due to irradiation of the secondary's atmosphere by the heated white dwarf primary star. This possibility might arise from the fact that the six mini-outbursts look like the rapid outbursts observed for ER UMa stars, a subclass of SU UMa stars which are thought to have mass transfer from the secondary stars on high rate (see also Osaki 1996; Kato et al. 1999). In fact, Hameury et al. (2000) made simulations of dwarf novae testing the effect of secondary illumination and disk illumination with several different parameters and commented that one of the simulated light curves looked like the observed rebrightening of EG Cnc. However, their light curve looks like that of damped oscillations which is quite different from the rebrightenings in EG Cnc. Furthermore, if the secondary star were illuminated, a periodic signal corresponding to the binary orbital period would have appeared. But no such evidence was known from observations. The disk illumination is also unlikely because the quiescent disk during six minioutbursts was rather cool as evident from its red color (Patterson et al. 1998).

The most unusual feature of the outburst of EG Cnc was that the star executed six mini-outbursts with a fairly constant level of maximum luminosity with more or less similar outburst intervals, but then abruptly went down into a deep faint minimum. In this paper, we address this particular feature of the light curve of EG Cnc by proposing a possible model in Sect. 2. We then present numerical simulations based on our simplifying model in Sect. 3 . Finally discussions are given in Sect. 4 and conclusions in Sect. 5 . 


\section{A working model}

\subsection{The standard disk instability model}

In our model the six mini-outbursts of EG Cnc are taken to be normal outbursts produced by the thermal disk instability. The disk instability model explains the dwarf nova outbursts as thermal relaxation oscillations between a hot fully ionized state and a cold unionized state in the bi-stable disk. The thermal relaxation oscillation is most easily understood locally in terms of the so-called Sshaped equilibrium curve. Figure 2a shows the hot upper and cool lower branch connected by the partially ionized unstable branch. At each radius in the disk there exist two critical surface densities, $\Sigma_{\max }$, above which no cold equilibrium state exists and $\Sigma_{\text {min }}$, below which no hot state exists. At the end of an outburst, all parts of the disk return back to a cold state and the surface density lies between $\Sigma_{\max }$ and $\Sigma_{\min }$ as illustrated schematically in Fig. 2b. Matter from the secondary and viscous evolution within the disk makes the surface density to increase in the disk. The surface density will ultimately reach the local critical density $\Sigma_{\max }$ at some point in the disk. A heating transition to the hot state results (see Fig. 2c). The heating front propagates both inward and outward, turning all of the disk matter into the hot state. During the hot state mass flows inward at a high rate and the surface density decreases. When $\Sigma_{\min }$ is reached the disk turns to the cool state. The cooling transition always occurs at the outer edge of the disk and the cooling front propagates inwards, extinguishing the outburst.

It is well known that for the outburst two different possibilities exist as to where the disk first reaches the ignition point, either at the outer edge or in the inner part of the disk. Accordingly two types of outbursts are known, the outside-in outburst and the inside-out outburst. An outside-in outburst results if the mass transfer rate from the secondary star is high while the inside-out outburst occurs if the viscous evolution within the disk is effective.

Rapid repetition of mini-outbursts with a recurrence time as short as seven days as observed after the end of the main outburst in EG Cnc can be understood due to either an increased mass transfer rate from the secondary star or to an enhanced viscosity in the disk. The model of Osaki et al. (1997) for EG Cnc was based on the second possibility.

Let us now describe our working model for the whole outburst cycle of WZ Sge stars, in general, and, EG Cnc, in particular, from the stand-point of temporal evolution of the disk viscosity. We assume that the viscosity in the hot state, $\alpha_{\text {hot }} \sim 0.2$, is produced by dynamo action by the MHD turbulence due to the "Balbus-Hawley instability", but in the cold quiescent disk these magnetic fields disappear due to poor conductivity as described in Sect. 1. The very low viscosity in quiescence in WZ Sge stars, $\alpha_{\text {cold }} \sim 0.001$, may then be produced by some other mechanisms such as tidal spiral density waves (see, e.g. Spruit 1989) or some other hydrodynamic instabilities (e.g. Papaloizou \& Pringle 1984). With such low viscosity the mass transferred from the secondary will be stored simply in a torus at the "Lubow and Shu radius" (Lubow \& Shu 1975), the circularization radius, and the mass accumulated will be very large when the next outburst is ignited. The outburst then is characterized by a large amplitude and long duration due to the large amount of mass accumulated during the long quiescence (see, e.g., Osaki 1995a).

\subsection{The effect of decreasing viscosity}

At the end of the outburst the viscosity will only gradually decrease due to the finite resistive decay time of the magnetic fields and the viscosity parameter may still remain high, as large as $\alpha_{\text {cold }} \sim 0.1$ in the cold state in the beginning quiescence.

Let us now consider what happens when the viscosity starts to decrease. If the viscosity in the cold disk would stay high all the time, the surface density will sooner or later reach the local critical density $\Sigma_{\max }$ at some point in the disk. and a normal outburst will be ignited as illustrated in Fig. 2. However if the viscosity decreases exponentially with time due to magnetic diffusivity, the critical surface density $\Sigma_{\max }$ increases exponentially since $\Sigma_{\max }$ is proportional to about $\alpha_{\text {cold }}^{-0.8}$. In this case a competition occurs between the local increase in surface density and the increase of the critical surface density, illustrated in Fig. 3. This situation is comparable to that of a runner who tries to reach the goal, but the goal is receding. If the runner wins, we have the ignition to normal outburst (Fig. 3a). If the runner once loses, he never makes it because the goal recedes increasingly faster. This is illustrated in Fig. 3b as the surface density curve is convex while the critical surface density curve is concave.

We further propose that the viscosity returns back to the original high value every time a mini-outburst occurs since the disk becomes hot again and fully ionized and the high electric conductivity is restored. The "BalbusHawley instability" becomes effective again, reproducing MHD turbulence and high viscosity. After the end of a mini-outburst, almost the same situation will be realized in the disk as before and a new competition begins. An additional fact influences the outcome if this competition: the dynamo generation of magnetic fields by the "BalbusHawley instability" is stochastic and in such a case the strength of regenerated magnetic fields and their configuration may not be the same after every outburst. The natural consequence of this is that there are some fluctuations in the resultant viscosity and its decay time fluctuates from mini-outburst to mini-outburst. If now the competition between the viscous diffusion of disk matter and the viscosity decay is very delicate, one time the diffusion of matter wins and an outburst occurs but another time the decay of viscosity wins. If then the increase of surface density fails to reach the critical one, it never catches up again since the viscosity decay is a run-away process and 


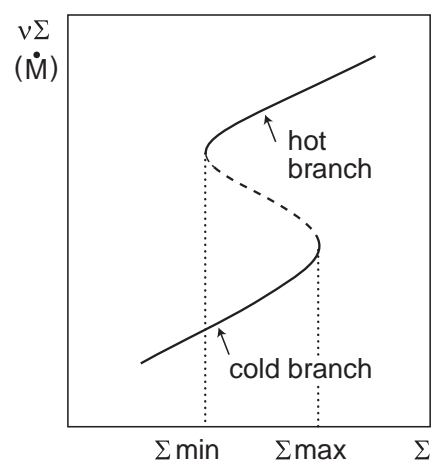

(a)

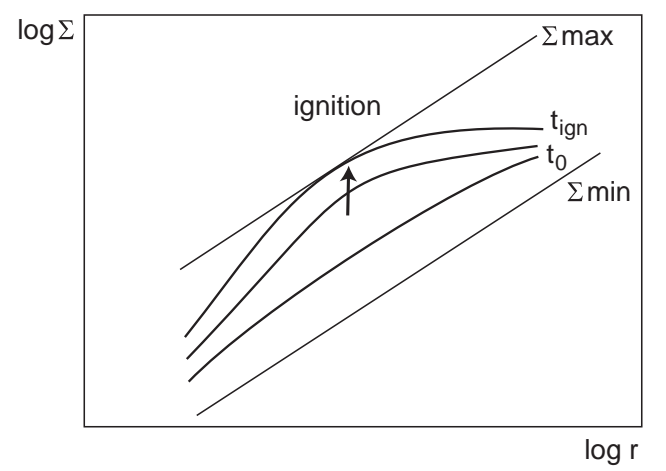

(b)

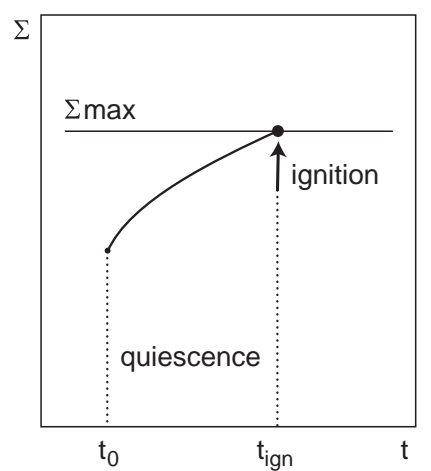

(c)

Fig. 2. a) A schematic S-shaped thermal equilibrium curve showing the two critical surface densities, $\Sigma_{\min }$ and $\Sigma_{\max }$. b) Time evolution of the surface density in the quiescent disk. c) Time evolution of surface density at the ignition point
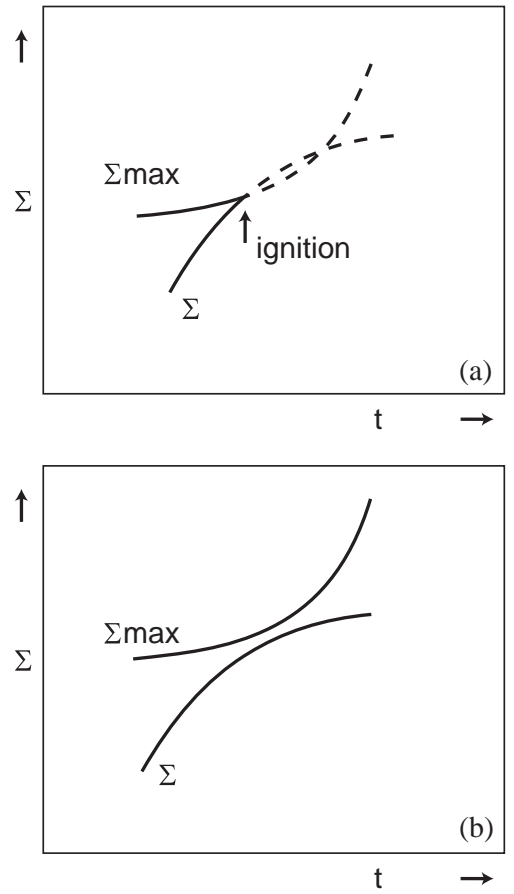

Fig. 3. Schematic diagrams for evolution of the surface density $\Sigma$ with time together with the critical surface density $\Sigma_{\max }$, which increases if a viscosity decay is taken into account. a) situation in a case where ignition occurs, b) when the critical surface density is not reached and no ignition occurs

the disk viscosity becomes very low and the star enters into a long phase of faint minimum. We postulate that the six such mini-outbursts are repeated in this way in EG Cnc before a final competition leads to a faint deep minimum.

We now estimate the characteristic decay time of magnetic fields and of the viscosity due to the finite conductivity in the cold disk matter. Hawley et al. (1996) found in numerical simulations that the dynamo created magnetic fields are no longer sustained and start to decay when the magnetic Reynolds number $R e_{\mathrm{m}}$ becomes less than $\approx 10^{4}$. Here the magnetic Reynolds number is $R e_{\mathrm{m}}=L v / \eta$ where $L, v$, and $\eta$ are the characteristic length scale, the characteristic velocity, and the magnetic diffusivity, respectively. We adopt $L \sim H$ and $v \sim c_{\mathrm{s}}$ in the accretion disk, where $H$ is the vertical scale height of the disk and $c_{\mathrm{s}}$ the isothermal sound velocity. For the decay time $\tau_{\text {decay }}$ of magnetic fields we then obtain

$\tau_{\text {decay }} \sim L^{2} / \eta \sim R e_{\mathrm{m}} \cdot H / c_{\mathrm{s}} \sim R e_{\mathrm{m}} \cdot \frac{1}{\Omega}$,

where $\Omega$ is the Keplerian rotation frequency of the disk matter. The magnetic Reynolds number in the cold disk is estimated to be $R e_{\mathrm{m}} \approx 10^{3}-10^{4}$ (Gammie \& Menou 1998). In the disks of WZ Sge stars the inverse of the Keplerian angular frequency is of the order of $300 \mathrm{~s}$. This yields the characteristic decay time of order of a week to a month. A time scale of this order seems to be right for our model, as the repetition time of mini-outbursts is about a week.

On the other hand, once the disk becomes hot and ionized, the "Balbus-Hawley instability" becomes effective and starts to build up the magnetic fields. Their growth time is thought to be very short, estimated to be of the order of the inverse of the Keplerian rotation frequency, $\tau_{\text {growth }} \sim 1 / \Omega$. This time-scale is sufficiently short that the decayed magnetic fields can be brought back to the original level and that thus the viscosity is refreshed to the original high value every time when a mini-outburst occurs.

This large disparity of growth rate and decay rate of magnetic fields is a main ingredient of our model. We now present the simulations based on these ideas.

\section{Numerical simulations}

We simulate the light curve of the six mini-outbursts and their sudden cessation in EG Cnc by means of a simplified model used for the simulation of the light curves of SU UMa stars (Osaki 1989) and WZ Sge (Osaki 1995a). Our model is based on a formulation by Anderson (1988) to treat time evolution of the disk radius in a dwarf nova outburst cycle. It was used extensively by one of the authors to simulate various light curves of SU UMa-type dwarf 
novae (Osaki 1989 for SU UMa stars in general; Osaki 1995a for WZ Sge; Osaki 1995b for ER UMa; Osaki 1995c for RZ LMi). The model disk consists of an inviscid disk component and a torus component. Since the disk component in our simplified model is taken as inviscid, only an outside-in type outburst can be treated. The outsidein outburst is triggered when the torus mass exceeds its critical value. We modify our previous calculations for the WZ Sge (Osaki 1995a) now taking into account a viscosity decay as described in the previous section.

The binary parameters used for EG Cnc in our simulations are those used for WZ Sge in the previous work (Osaki 1995a): (1) the mass of the primary white dwarf, $M_{1}=1.0 M_{\odot} ;(2)$ the radius of the white dwarf, $R_{1}=$ $0.610^{9} \mathrm{~cm}$; (3) the mass of the secondary star, $M_{2}=$ $0.1 M_{\odot} ;$ (4) the binary separation, $A=4.4610^{10} \mathrm{~cm}$; (5) the circularization radius or Lubow-Shu radius, $r_{\mathrm{LS}}=$ $0.23 A=10^{10} \mathrm{~cm}$. We take a mass transfer rate $\dot{M}=$ $1.510^{15} \mathrm{gs}^{-1}$.

In order to explain the long quiescence of EG Cnc, 19 years, we assume that the quiescent disk viscosity is extremely low, $\alpha_{\text {cold }} \sim 0.001$. In our simplified model, the viscosity appears through the quantity $\beta$,

$\beta=\left(\Delta r / r_{\mathrm{t}}\right)\left(\Sigma_{\max } / \Sigma_{\text {cool }}\right)$

where $r_{\mathrm{t}}$ is the torus radius, i.e., the radius of the disk's outer edge and $\Delta r$ the radial width of the torus. $\Sigma_{\max }$ is the critical maximum surface density and $\Sigma_{\text {cool }}$ the surface density of the cold disk just after the end of the outburst, estimated to be $\Sigma_{\text {cool }} \simeq 2 \times \Sigma_{\text {min }}$ (for more details see Osaki 1989). Therefore, a low viscosity value, $\alpha$, corresponds to a large $\Sigma_{\max }$ and therefore to a large $\beta$. It may be noted here that we used $\beta=0.6$ to simulate VW Hyi (Osaki 1989) while $\beta=40$ was used to simulate the long quiescence of WZ Sge (Osaki 1995a). Similarly to explain the long quiescence of EG Cnc we adopted $\beta=20$ for the pre-outburst cold disk. The light curve of the main outburst of EG Cnc is calculated in a similar way as that for WZ Sge (Osaki 1995a).

For the post-outburst rebrightening of EG Cnc, we now incorporate the viscosity decay described in the previous section into our simplified model. In order to accommodate the exponential decay of viscosity, we take the quantity $\beta$ not constant in time, but exponentially increasing in the following way

$\beta=\beta_{0} \exp (t / \tau)$

where $\beta_{0}$ is an initial value of $\beta$, and $\tau$ is a characteristic time for the viscosity decay, of the order of a week to a month, and $t$ is the time counted from the end of either the main outburst or each mini-outburst. That is, viscosity is assumed to be refreshed to a high value each time a minioutburst occurs. In this paper, the quantities $\beta_{0}$ and $\tau$ are two adjustable parameters to reproduce the sequence of rapid mini-outbursts with a mean recurrence time of seven days. This indicates a fairly high viscosity and requires $\beta_{0}$ to be small.
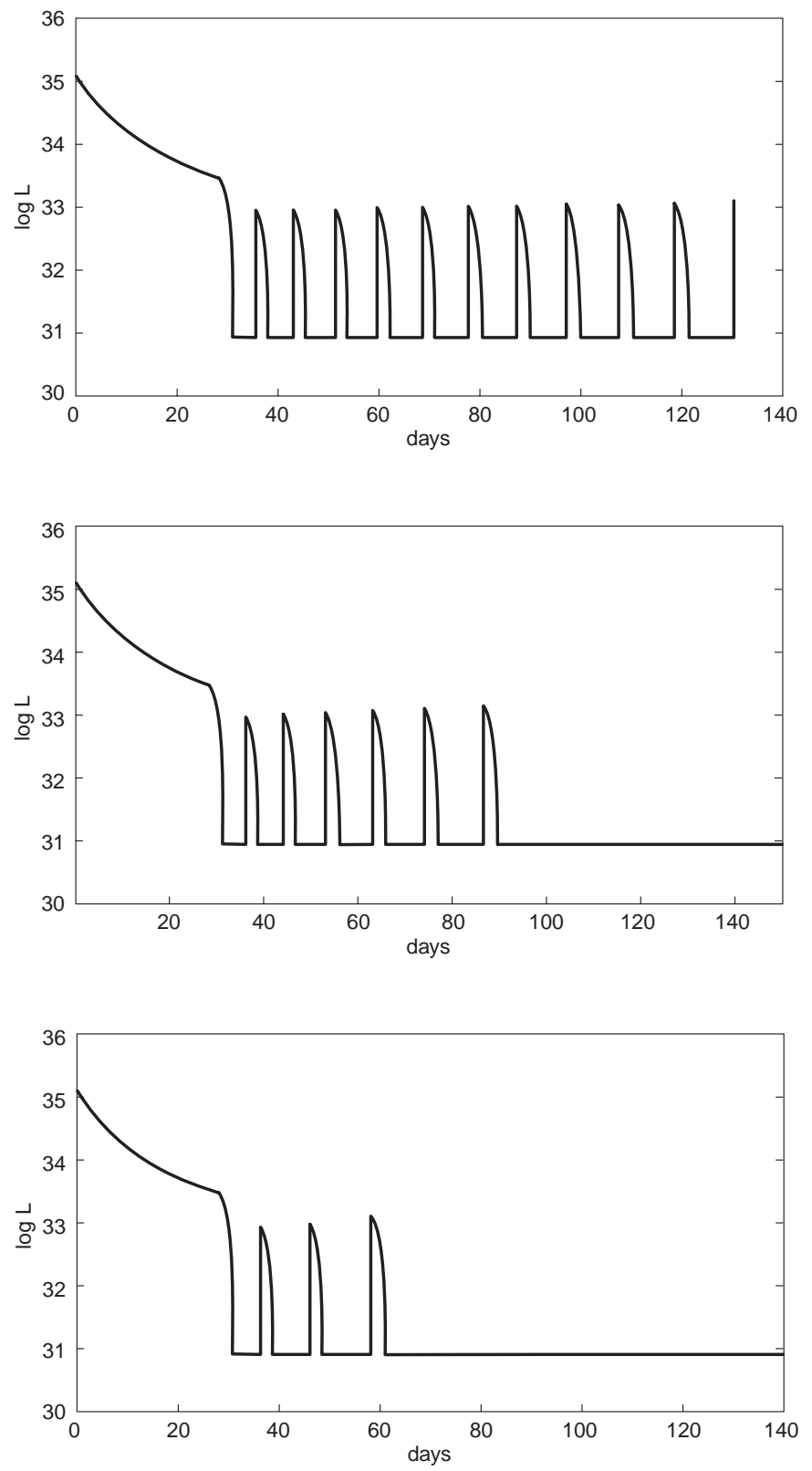

Fig. 4. Light curve simulations based on the simplified model, for a case with $1 \%$ increase in $\beta_{0}$ after each mini-outburst (top panel), for $2 \%$ increase (middle panel) and for $4 \%$ increase (bottom panel)

We have varied the parameter values for these two quantities and found that $\beta_{0}=0.08$ and $\tau=25$ days reproduce the observed recurrence time of 7 days (other choices may also be possible). If with each mini-outburst the viscosity in the cold disk is refreshed to the original high value the mini-outbursts continue to repeat. This can be seen from the full simulations with all parameters kept the same and with enhanced viscosity in the cold disk (Osaki et al. 1997). However as discussed in the previous section, there will be fluctuations in strength and configuration of magnetic fields which are regenerated during each mini-outburst. In order to account for such stochastic fluctuations in magnetic fields and therefore in the resultant viscosity, we have performed simulations with secular 
variations of $\beta_{0}$ and $\tau$. For instance, $\beta_{0}$ is not returned back to the same value but a small increase is allowed after each mini-outburst. Figure 4 illustrates three light curves calculated for an increase of the value $\beta_{0}$ by $1 \%, 2 \%$, and $4 \%$ relative to the previous value when a new minioutburst occurred. As seen in Fig. 4, top panel, more than ten mini-outbursts occur in sequence. With $2 \%$ increase of $\beta_{0}$ an abrupt cessation after the sixth outburst occurs (middle panel). The most important feature of these calculations is that a different cessation of outbursts occurs for an even extremely small difference of the parameters. The light curve shown in the middle panel of Fig. 4 with its six consecutive mini-outbursts and their sudden end is very similar to that observed in EG Cnc. This sensitive dependence of the number of mini-outbursts on the parameter $\beta_{0}$ suggests that the exact number of mini-outbursts is a matter of chance and can be different after another superoutburst. Note that the bolometric luminosity shown in Fig. 4 is due to radiation from the hot disk plus that from the stream impact, and no contributions are included either from the secondary star, or the central white dwarf, or the cold disk.

This phenomenon of sudden cessation is most easily understood from Fig. 5 where the evolutions of the torus mass and of its critical value are shown in the same diagram for the case of $2 \%$ increase. Each time the torus mass reaches its critical value a thermal instability is triggered and the disk jumps to a hot state. Six times the increase of torus mass was fast enough to reach the critical torus mass and outbursts were ignited. However in the last case these two curves came close to each other but nearly missed contact and diverged afterwards. This explains a sudden cessation of outbursts in our calculations.

Similar calculations have been performed by slightly reducing the characteristic time $\tau$ after each mini-outburst and we have obtained similar results to those shown above. We expect to also get similar results if we decrease the mass supply rate slightly after each mini-outburst because then it becomes more and more difficult to reach the critical surface density (see discussion Sect. 4.2).

\section{Discussion}

\subsection{The quiescent luminosity of $E G C n c$}

Further support for viscosity decay comes from the decrease of the quiescent luminosity from 16 to $18 \mathrm{mag}$ within one or two months after the cessation of the minioutbursts of EG Cnc (Patterson et al. 1998, compare Fig. 1). This decrease in quiescent luminosity is most naturally interpreted as caused by a decrease of luminosity of the cold disk. We first note that most light in minimum of the mini-outbursts comes from the cold disk and not from the central white dwarf because in quiescence during mini-outbursts the color is very red (Patterson et al. 1998). A decrease in luminosity after the end of the rapid mini-outbursts thus indicates a decrease in luminosity of the cold disk, in accordance with our model.
Since the radiative flux, $F$, from the surface of an accretion disk in thermal equilibrium is given in terms of viscous stresses in the standard accretion disk by

$2 F=\frac{9}{4} \nu \Sigma \Omega=\frac{3}{2} \alpha_{\text {cold }} \Omega \int P \mathrm{~d} z$,

we can write for the quiescent disk luminosity, $L_{\text {disk }}$,

$$
\begin{aligned}
L_{\text {disk }} & =\int(2 F) \mathrm{d} S=\iint \frac{3}{2} \alpha_{\text {cold }}(\Re / \mu) T \Omega \rho \mathrm{d} z \mathrm{~d} S \\
& \simeq \alpha_{\text {cold }} \frac{3}{2}(\Re / \mu)\langle T\rangle\langle\Omega\rangle M_{\text {disk }}
\end{aligned}
$$

where $\mathrm{d} S$ is the surface element, $P, \nu, \rho, T, \Re, \mu, M_{\text {disk }}$ are pressure, kinematic viscosity, density, temperature, gas constant, mean molecular weight of the gas in the cold disk, and disk mass, respectively. Quantities within brackets are mean values over the disk. In our light curve simulations presented in the previous section, no contribution from the cold disk is taken into account, thus we have to add its contribution to the luminosity of the star in quiescence. Since almost no accretion occurs from the disk to the central star during quiescence, the mass in the disk taken in the equation above should not decrease. The decrease of luminosity from 16 mag to 18 mag after the end of the mini-outbursts therefore may result from a decrease of the quantity $\alpha_{\text {cold }}\langle T\rangle$. Since the disk temperature goes down with a decrease of viscosity, the viscosity parameter, $\alpha_{\text {cold }}$, must have decreased during this interval pointing to even lower conductivity.

\subsection{A special feature of WZ Sge stars}

Finally, let us address the question why the post-outburst rebrightening phenomenon occurs most often in WZ Sge stars. This question was addressed before by Kato et al. (1997) and the following possibility and the observational evidence supporting it were already put forward by these authors. We essentially follow their line. One of the distinguishing characters of WZ Sge stars as compared with other SU UMa stars is the large amount of mass accumulated in the disk during the long quiescence. This leads to their outstanding large-amplitude and long-duration outbursts. This will also bring substantially more mass beyond the $3: 1$ resonance radius in order to transfer the large amount of angular momentum released during accretion. If a fraction of this is left over when the cooling transition begins to propagate and the main outburst ends, its viscous relaxation during the beginning quiescence will feed mass from the outer disk inward at an initially higher but gradually decreasing rate. This could well be instrumental to bring about the initial reflaring in the way discussed in this article, making it peculiar to WZ Sge stars. Evidence for this may be seen in the observation of superhumps in the early quiescence in EG Cnc (Kato et al. 1997; Patterson et al. 1998) pointing to a reservior of mass beyond the 3:1 resonance radius, and in the decreasing level of the disk light during the quiescence in between the sequence of mini-outbursts (Fig. 1), indicating the gradual depletion of this reservoir of additional mass supply. 


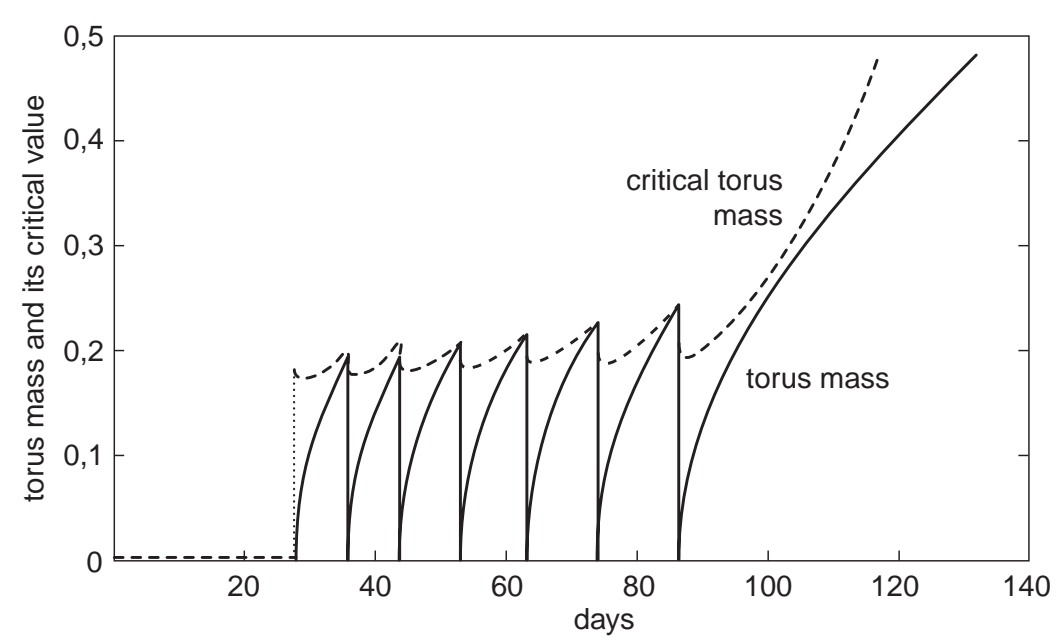

Fig. 5. Evolution of the torus mass after the superoutburst, together with that of the critical mass in the torus for a $2 \%$ increase in $\beta_{0}$ (correspondingly decreasing viscosity) after each mini-outburst, same calculation as shown in the middle panel of Fig. 4

\section{Conclusions}

Already in earlier modeling of the light curve of EG Cnc (Osaki et al. 1997) the six mini-outbursts were reproduced in good agreement with the observations. In these calculations the viscosity in the cold disk was kept artificially high during the consecutive mini-outbursts, but was assumed to drop to an extremely small value after the end of the sixth mini-outburst. These viscosity values were taken ad hoc to fit the observations, without a specific model for such changes. The introduction of magnetic field decay now provides a reason for the gradual change of viscosity from the high value in the hot state to the lower and finally very low value in quiescence. These considerations constitute a physical justification for the assumptions made by Osaki et al. (1997). Those more detailed calculations can otherwise be seen as a confirmation of the now performed work based on a simplified model.

In the present investigation we have proposed a model for the consecutive mini-outbursts of EG Cnc and their abrupt cessation based on the decay of the small-scaledynamo produced MHD turbulent viscosity due to finite conductivity of the cold disk matter. We have successfully simulated the light curve of EG Cnc using a simplified model that can treat only the outside-in outburst, that is, outbursts occur when the torus reaches its critical value. In reality the ignition of mini-outbursts in EG Cn might occur most likely in the middle part and not necessarily near the outer edge of the disk. In such a case, what was summarily called "mass supply from the secondary star" in our simulations may be interpreted as mass supply by viscous diffusion from the outer disk to the ignition point.

Should this model presented here prove correct it lends strong support to the theory of magnetohydrodynamic turbulence as cause of the viscosity in high temperature accretion disks. It also supports again the notion of very low viscosity for the quiescent disks in WZ Sge stars as compared to that of ordinary dwarf novae. In this and in other features not discussed here in detail it fits well into the gradually emerging solution of the puzzle of accretion disk viscosity.

Acknowledgements. Yoji Osaki acknowledges financial support from the Japanese Ministery of Education, Science, Sports and Culture with a Grants-in Aid for Scientific Research No. 12640237.

\section{References}

Anderson, N. 1988, ApJ, 325, 266

Armitage, P. J. 1998, ApJ, 501, L189

Bailey, J. 1979, MNRAS, 189, 41p

Balbus, S. A., \& Hawley, J. F. 1991, ApJ, 376, 214

Balbus, S. A., \& Hawley, J. F. 1998, Rev. Mod. Phys., 70, 1

Brandenburg, A., Nordlund, A., Stein, R. F., et al. 1995, ApJ, 446, 741

Cannizzo, J. K. 1993, in Accretion Disks in Compact Stellar Systems, ed. J. C. Wheeler (Singapore, World Scientific), 6

Chandrasekhar, C. 1961, in Hydrodynamic and Hydrodynamic Stability (Oxford University Press, Oxford), 1981

Downes, R. A. 1990, AJ, 99, 939

Gammie, C. F., \& Menou, K. 1998, ApJL, 492, L75

Hameury, J.-M., Lasota, J.-P., \& Warner, B. 2000, A\&A, 353, 244

Hawley, J. F., Gammie, C. F., \& Balbus, S. A. 1996, ApJ, 464, 690

Howell, S. B., DeYoung, J. A., Mattei, J. A., et al. 1996, AJ, 111,2367

Howell, S. B., Szkody, P., \& Cannizzo, J. K. 1995, ApJ, 439, 337

Kato, T., Nogami, D., Baba, et al. 1996, PASJ, 48, L21

Kato, T., Nogami, D., Matsumoto, K., et al. 1997, preprint Superhumps and Repetitive Rebrightenings of the WZ Sge-type Dwarf nova, EG Cancri,

ftp://ftp.kusastro.kyoto-u.ac.jp/pub/vsnet/ preprints $/ \mathrm{EG}_{\mathrm{C}} \mathrm{nc} /$

Kato, T., Nogami, D., Baba, et al. 1999, in Disk Instabilities in Close Binary Systems, ed. S. Mineshige, \& J. C. Wheeler (Universal Academy Press, Tokyo), 45

Lubow, S. H., \& Shu, F. H. 1975, ApJ, 198, 383 
Matsumoto, K., Kato, T., Ayani, T., et al. 1998a, Commissions 27 and 42 of the IAU Information Bulletin on Variable Stars, No. 4613

Matsumoto, K., Nogami, D., Kato, T., et al. 1998b, PASJ, 50, 405

Meyer, F., \& Meyer-Hofmeister, E. 1999, A\&A, 341, L23

Meyer-Hofmeister, E., Meyer, F., \& Liu, B. F. 1998, A\&A, 339, 507

O'Donoghue, D., Chen, A., Marang, F., et al. 1991, MNRAS, 250,363

Osaki, Y. 1989, PASJ, 41, 1005

Osaki, Y. 1994, in Theory of Accretion Disks-2, ed. W. Duschl et al. (Kluwer Academic Publishers, Dordrecht), 93

Osaki, Y. 1995a, PASJ, 47, 47

Osaki, Y. 1995b, PASJ, 47, L11

Osaki, Y. 1995c, PASJ, 47, L25

Osaki, Y. 1996, PASP, 108, 39
Osaki, Y., Shimizu, S., \& Tsugawa, M. 1997, PASJ, 49, L19, Paper I

Papaloizou, J. C. B., \& Pringle, J. E. 1984, MNRAS, 208, 721

Patterson, J., Augusteijn, T., Harvey, D. A., et al. 1996, PASP, 108,748

Patterson, J., Kemp, J., Skilman, D. R., et al. 1998, PASP, 110,1290

Patterson, J., McGraw, J. T., Coleman, L., \& Africano, J. L. 1981, ApJ, 248, 1067

Richter, G. A. 1992, in Vina del Mar Workshop on Cataclysmic Variable Stars, ed. N. Vogt, ASP Conf. Ser., 29, 12

Shakura, N. I., \& Sunyaev, R. A. 1973, A\&A, 24, 337

Spruit, H. 1989, in Theory of Accretion Disks, ed. F. Meyer et al. (Kluwer Academic Publishers, Dordrecht), 325

Smak, J. 1984, Acta Astron., 34, 161

Smak, J. 1993, Acta Astron., 43, 101

Velikov, S. 1959, J. Expl. Theoret. Phys. (USSR), 36, 1398 